\title{
Measuring Inequality Change in an Economy with Income Growth
}

\author{
Gary S. Fields* \\ Cornell University
}

This paper analyzes how to measure changes in inequality in an economy with income growth. The discussion distinguishes three stylized kinds of economic growth:

1. high income sector enrichment,

2. low income sector enrichment,

3. high income sector enlargement, in which the high income sector expands and absorbs persons from the low income sector.

The two enrichment types pose no problem for assessing inequality change in the course of economic growth: for high income sector enrichment growth, inequality might reasonably be said to increase, whereas for low income sector enrichment, inequality might be said to decrease. These judgments are non-controversial and non-problematical. Where problems arise is in the case of high income sector enlargement growth. In that case, the two alternative approaches have been shown in this paper to yield markedly different results:

1. The traditional inequality indices generate an inverted-U pattern of inequality. That is, inequality rises in the early stages of high income sector enlargement growth and falls thereafter.

2. The new approach suggested here, based on axioms of gap inequality and numerical inequality, generates a $U$ pattern of inequality. That is, inequality falls in the early stages of high income sector enlargement growth and rises thereafter.

The discrepancy between the familiar indices and the alternative approach based on axioms of gap inequality and numerical inequality bears further scrutiny. Two courses of action are possible. One might try to axiomatize inequality in ways that generate an inverted-U pattern in high income sector enlargement growth, thereby rationalizing the continued use of the usual inequality indices with the inverted-U property. Alternatively, one might retain the axioms proposed here, embed them into a more formal structure, and construct a family of inequality indices consistent with them. Others might wish to pursue the first course; I am at work on the second.

\footnotetext{
${ }^{*}$ An earlier version of this paper was presented at the Econometric Society Fifth World Congress, Cambridge, Massachusetts, and at seminars at Cornell, Warwick, and Southampton Universities. I have benefited from the comments of seminar participants and anonymous referees.
} 


\section{Statement of the problem}

When economic growth takes place, a new income distribution vector is obtained. It is customary for applied researchers to summarize the data by changes in such statistics as level of national income (GNP), relative income inequality, and absolute poverty. A vast empirical literature on the subject has appeared, dating from Kuznets' justly famous 1955 paper. Besides the many studies of individual countries, multi-country studies appear in the works of Ahluwalia (1976), Chenery (1979), Fields (1980) and Loehr and Powelson (1981), among others.

This paper addresses the relative inequality aspect, taking for granted that the change in inequality is a topic of interest. I am concerned with a methodological matter that underlies empirical investigations of inequality change in the course of economic growth, namely: what criterion to use to gauge whether inequality increases or decreases when economic growth occurs. ${ }^{1}$

What is done in practice is this. Empirical researchers use inequality measures that are consistent with the Lorenz criterion in the weak sense. This means that when one income distribution Lorenz-dominates another, the first distribution is deemed at least as equal as the second. ${ }^{2}$ The class of Lorenz-consistent inequality indices excludes certain inequality measures that have been proposed (e.g., the variance and the Kuznets ratio ${ }^{3}$ ) but includes many others - among them, the coefficient of variation, the Gini coefficient, the Atkinson index and the Theil index. In an empirical situation, if several such Lorenz-consistent unequality indices are calculated and if inequality rises/falls by all of these measures, empirical researchers usually feel safe in concluding that inequality has in fact risen/fallen.

Making inequality comparisons on the basis of Lorenz-consistent indices reflects certain judgments about the very nature of inequality: ${ }^{4}$

Al. Axiom of scale irrelevance: If one income distribution is a scalar multiple of another, then the two distributions are equally unequal.

A2. Axiom of anonymity (or symmetry): If one income distribution is a permutation of the other, then they are equally unequal.

A3. Axiom of population replication: If two populations differ in size but the income shares of the corresponding percentile groups are the same, then the two distributions are equally unequal.

A4. Axiom of rank-preserving equalization: If one distribution $X$ is obtained from another distribution $Y$ by a rankpreserving equalization of income, then $X$ is more equal than $Y .^{5}$

\footnotetext{
${ }^{1}$ Also of interest is inequality change during economic contractions. These cases, fewer in number, may be thought of as negative growth and may be analyzed similarly.

${ }^{2}$ A more stringent criterion, 'strong Lorenz consistency', would require that the first distribution be regarded as strictly more equal than the second. An example of an index which satisfies only the weaker criterion is the income share of the poorest or richest X\%. Income changes among the remaining 1 - X\% may lead to Lorenz-domination, yet the share received by the poorest or richest $\mathrm{X} \%$ is unchanged.

${ }^{3}$ The Kuznets ratio is defined as $\mathrm{K}=\sum\left|\theta_{i}-1 / n\right|$, where $\theta_{i}$, is the income share of the ith individual and $\mathrm{n}$ is the number of individuals in the population.

${ }^{4}$ The modem demonstration of the correspondence between Al, A2 and A4 and the Lorenz criterion for the case of fixed population size is due to Fields and Fei (1978). The extension to variable population sizes is immediate, given A3.

${ }^{5} \mathrm{~A}$ rank-preserving equalization is a transfer of income from a relatively rich person to a relatively poorer one holding their ranks in the income distribution constant.
} 
Based on these axiomatic judgments, the Lorenz criterion for inequality comparisons is justified. It states that when one income distribution Lorenz- dominates another, the first distribution is more equal than the second. The Lorenz criterion and the axiomatic justification underlying it command wide support among economists and other social scientists.

The Lorenz-consistent inequality indices, although they accept the Lorenz criterion, go well beyond it. The Lorenz criterion provides just a partial ordering; it ranks income inequality only for distributions whose Lorenz curves do not intersect. When two distributions' Lorenz curves cross, the Lorenz criterion provides no inequality ranking; such pairs of distributions are Lorenz-incomparable. ${ }^{6}$ By contrast, the Lorenz-based inequality indices are complete; they provide inequality rankings for all pairs of income distributions. Each such inequality index completes the ordering in a particular way. The completion rule is implicit in the choice of the index itself.

Inequality indices have been axiomatized by authors including Atkinson (1970), Sen (1973), Kondor (1975), Kolm (1976), Cowell (1977), Cowell and Kuga (1981), Blackorby and Donaldson (1978), Kakwani (1980) and Shor- rocks (1980, 1983). For instance, an axiom of concern with income ranks would justify use of the Gini coefficient whereas a concern with decomposability into within-group and between-group inequality helps justify the Theil index. A comprehensive review of this literature is to be found in Foster (1985). The point I would note from my reading of this literature is that these axioms for completing the ordering by use of a particular index have not been derived in a growth context. Maybe these indices are suitable for gauging inequality change in an economy with income growth, maybe not; but from the available justifications, we have no way of knowing.

The question to which this paper is addressed is: How suitable are these indices for measuring how income inequality changes in a growing economy? My strategy for answering this question is to posit several stylized types of economic growth. I then set forth what 'should' be happening to income inequality if growth proceeds in the posited ways. Next, I investigate how the standard indices treat each stylized growth type. If the treatment were found to accord with what 'should' happen to inequality in that growth type, we could rest assured that the standard measures are appropriate for determining how inequality changes under different patterns of income growth. But this is not what I find. Instead, for one very important type of economic growth, the standard treatment conflicts with what 'should' happen. To try to resolve the problem, I evaluate possible ways suggested by others for completing the inequality ordering in an economy with income growth. Upon finding that the suggested approaches do not satisfactorily resolve the issue, I then outline the start of an alternative approach and demonstrate the existence of an index consistent with the posited axiomatic structure.

\section{Inequality change in several stylized growth types}

All of the essential points of this paper can be analyzed by considering an economy with two real incomes - a high income of $\$ 4$ and a low income of $\$ 1$ - and a constant population of six persons. Let the initial income distribution vector be $A=(1,1,1,1,1,4)$. Suppose the economy experiences income growth of $\$ 3$, which might be distributed in three

\footnotetext{
${ }^{6}$ The term 'Lorenz-incomparable' is something of a misnomer. The two distributions can be compared. It's just that the Lorenz criterion is incapable of ranking them.
} 
alternative ways:

\section{'High income}

sector enrichment': $\quad A=(1,1,1,1,1,4) \rightarrow B=(1,1,1,1,1,7)$,

'Low income

sector enrichment': $A=(1,1,1,1,1,4) \rightarrow C=(1.6,1.6,1.6,1.6,1.6,4)$,

'High income

sector enlargement': $\quad A=(1,1,1,1,1,4) \rightarrow D=(1,1,1,1,4,4)$.

These are stylized versions of three different patterns: respectively, the rich getting richer while the poor get nowhere, the poor benefiting uniformly while the rich remain stationary, and some of the poor moving up while others of the poor remain behind. While no country's growth experience is represented exactly by any of these patterns, empirical studies by development economists would certainly regard each pattern as a stylized typology relevant to some circumstances. Particularly appropriate is the 'high income sector enlargement' type of growth. In the words of Fei and Ranis (1964, p. 7): 'The heart of the development problem may be said to lie in the gradual shifting of the center of gravity of the economy from the agricultural to the industrial sector ... gauged in terms of the reallocation of the population between the two sectors in order to promote a gradual expansion of industrial employment and output.' Similar characterizations appear in the work of Lewis $(1954)$ and Kuznets $(1955,1966)$.

How 'should' inequality change in each of these examples? First consider high income sector enrichment, as in the change from $A$ to $B$. One sees intuitively that inequality is rising. In fact, the new income distribution is Lorenz-inferior to the old; see fig. la. Hence, by the Lorenz criterion or by a Lorenz-consistent inequality index:

C1. Inequality increases for high income sector enrichment (e.g., when the income distribution changes from $A$ to $B$ ).

By similar reasoning, in low income sector enrichment, as in the change from $A$ to $C$, one sees intuitively that inequality is falling, the new income distribution being Lorenz-superior to the old (fig. 1b). Thus: 
(a)

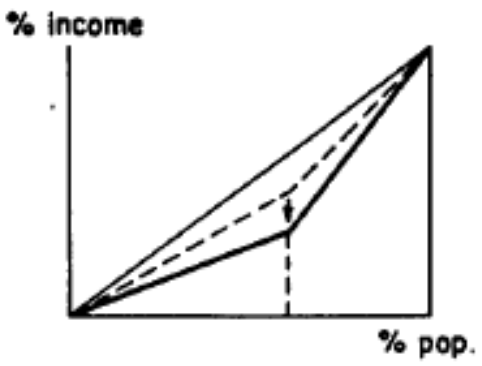

(b)

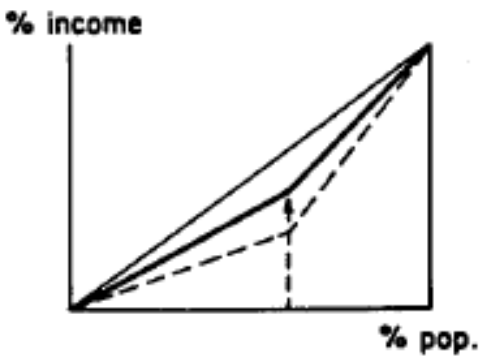

(c)

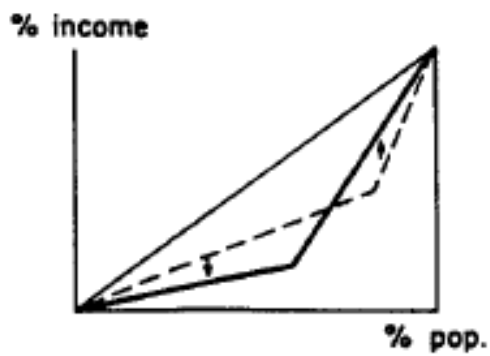

Fig. 1. Lorenz curves for three types of income growth. Original Lorenz curves are denoted by dashed lines, new Lorenz curves by bold lines. (a) High income sector enrichment, (b) Low income sector enrichment, (c) High income sector enlargement.

C2. Inequality decreases for low income sector enrichment (e.g., when the income distribution changes from $A$ to $C)$.

What about the change in high income sector enlargement, as from $A$ to $D$ ? Just looking at the numbers, it is not apparent what to say about how inequality changes. Furthermore, the Lorenz criterion is not helpful, because the Lorenz curves necessarily cross; see fig. 1c. ${ }^{7}$ Nonetheless, it seems desirable to say something rather than remain silent on the issue. ${ }^{8}$ And it is clear that what we say will depend on the division of the population between the different income categories.

Thus:

C3. Inequality changes according to the numbers of persons in the two groups in the case of high income sector enlargement (e.g., when the income distribution changes from $A$ to $D$ ).

The task before us is to specify how inequality depends on the numbers in the high and low income categories.

There are several ways of doing this. One is to accept the pattern generated by commonly-used inequality indices,

\footnotetext{
${ }^{7}$ The rationale for this and other Lorenz curve shifts is to be found in Fields (1979). In this case, the income shares of the richest and poorest persons both increase. This means that where the Lorenz curve emanates from the lower left origin, the new curve lies below the old, and where it emanates from the upper right origin, the new curve lies above the old. The two curves cross in the middle.

${ }^{8}$ That is, leaving the matter as a partial ordering and not ranking the inequality of distributions like $A$ and $D$ does not seem to be an acceptable option.
} 
which go beyond the Lorenz partial ordering by completing the inequality rankings in particular ways. A second is to examine the income distribution vectors, determine what we 'see' in the data, and judge inequality change accordingly. A third is to develop an axiomatic foundation for determining how inequality changes when there is income growth of the specified type. These courses are pursued below and are shown to yield conflicting verdicts.

\section{Approaches to inequality change in high income sector enlargement}

\subsection{What the commonly-used inequality measures show}

A number of researchers have examined how different inequality indices change in the course of high income sector enlargement growth. In his classic paper, Kuznets (1955) produced a number of examples demonstrating that income inequality measured by income shares of particular percentile groups first increases, then decreases, producing an inverted-U pattern. Subsequent research demonstrated that the emergence of an inverted-U was hardly coincidental. Knight (1976) and Fields (1979) showed independently that in the case of two incomes (high and low), the Gini coefficient necessarily follows an inverted- $U$ with an interior maximum. Swamy (1967) showed an inverted-U for the coefficient of variation. Robinson (1976) showed that the log variance is a quadratic form increasing at a decreasing rate; in the case of zero within-sector inequality, his mathematics imply that inequality follows an inverted-U pattern. Anand and Kanbur (1984) considered a number of other measures (Theil's entropy index, Theil's second measure, Atkinson's index, and the squared coefficient of variation) in addition to the Gini coefficient and the log variance. Their results imply that in the case of zero within-sector inequality, all of the indices considered must follow an inverted- $U$ pattern.

Table 1 displays the high income sector enlargement process, embodying the change from $[1,1,1,1,1,4]$ to $[1,1,1,1,4,4]$ described earlier. Three commonly-used inequality indices - the income share of the poorest, the Gini coefficient and the log variance - are calculated. All exhibit the inverted-U pattern.

Table 1

The inverted-U inequality pattern in a high income sector enlargement process.

\begin{tabular}{|c|c|c|c|}
\hline $\begin{array}{l}\text { Income } \\
\text { distribution } \\
\text { (1) }\end{array}$ & $\begin{array}{l}\text { Income } \\
\text { share of } \\
\text { poorest } 50 \% \\
\text { (2) }\end{array}$ & $\begin{array}{l}\text { Gini } \\
\text { coefficient } \\
\text { (3) }\end{array}$ & $\begin{array}{l}\text { Log } \\
\text { variance } \\
\text { (4) }\end{array}$ \\
\hline$(1,1,1,1,1,1)$ & $50.0 \%$ & 0.000 & 0.000 \\
\hline$(1,1,1,1,1,4)$ & 33.3 & 0.278 & 0.267 \\
\hline$(1,1,1,1,4,4)$ & 25.0 & 0.333 & 0.427 \\
\hline$(1,1,1,4,4,4)$ & 20.0 & 0.300 & 0.480 \\
\hline$(1,1,4,4,4,4)$ & 33.3 & 0.222 & 0.427 \\
\hline$(1,4,4,4,4,4)$ & 42.8 & 0.118 & 0.267 \\
\hline$(4,4,4,4,4,4)$ & 50.0 & 0.000 & 0.000 \\
\hline
\end{tabular}

"Inequality varies inversely with the income share of the poorest. 
The only rationale I have heard for the inverted-U pattern is circular - that inequality increases and then decreases, because that's what many inequality measures do. Such justifications for certain of these indices as sensitivity to income ranks or decomposability do not suffice for purposes of assessing inequality change in the course of economic growth. To the best of my knowledge, no other rationale for the inverted-U in this process has been offered.

\subsection{A look at the income distribution vectors}

One way of determining how inequality should change in high income sector enlargement is to examine the income distribution vectors themselves. Start with the early phases of a high income sector enlargement process.

Imagine a situation in which one person is rich and everyone else is poor. Suppose that as a result of economic growth, a second person (one who was previously poor) is enabled to become rich, the incomes among everyone else remaining unchanged, e.g., $[1,1,1,1,1,4] \rightarrow[1,1,1,1,4,4]$, as above. What happens to income inequality? Most economists to whom I have asked this question answer intuitively that income inequality has declined. Their reasoning goes roughly along the lines that the rich person is now in a less elite position, and that for a given gap in incomes between those who are rich and those who remain poor, the less elite position of the rich acts to reduce inequality.

Now imagine another situation in which the initial distribution of income is quite different: most of the people in the economy are rich but two are poor. Suppose now that economic growth enables one of the two who had originally been poor to become rich while, as before, incomes among others in the economy remain unchanged. For example, suppose the income distribution goes from $[1,1,4,4,4,4]$ to $[1,4,4,4,4,4]$, How does income inequality change in this case? Again, on a purely intuitive level, most economists to whom I have asked this question say that income inequality has increased. They see in this change a situation in which the remaining poor person has now become more isolated from others in the economy. In the minds of these observers, income inequality increases on that account.

Think now of a sequence leading from the first situation to the second, as illustrated by column (1) of table 1. That is, start with a small number of high income persons and allow economic growth to proceed in such a way that the high income sector enlarges to accommodate an ever-increasing share of the total population. Allow economic growth to proceed in this way for a long time, so that fewer and fewer people are left behind in the low income sector, and eventually we reach a situation where few people are in the low income sector and economic growth reduces their numbers still further. By the preceding reasoning, income inequality should decrease in the early stages of a growth process of this type and increase in the later stages. It is quite plausible that somewhere in between, inequality reaches an interior minimum. In the absence of arguments to the contrary, suppose for now that inequality declines monotonically until the minimum and increases monotonically thereafter. This intuition suggests that inequality follows a $U$ pattern in this type of economic growth - contrary to what is generated by the standard inequality measures.

\subsection{A formulation of numerical inequality}

The high income sector enlargement type of growth process is characterized by a constant income gap between the 
high and low income sectors but changing numbers in the two groups. If we conceptualize total inequality as depending upon the intersectoral income gap and the numbers in each, then with a constant income gap, inequality in this growth process varies as does numerical inequality.

How does numerical inequality vary? In the case where a population consists of two groups, it may be very reasonable to regard numerical inequality as being minimized when the two groups are of equal size. In this sense, a family of two sons and two daughters may be regarded as having a more equal sex distribution of offspring than a family with four children of one sex. In addition, the further is the actual distribution from fifty-fifty, the more numerical inequality there may be said to be. One blonde in a group of brunettes, one nudist amidst clothed bathers, one cat in a group of dogs - all are situations of pronounced numerical inequality. By analogy, when there are two fixed income amounts, numerical equality might be said to be greatest when half the people receive one income and half the other and to be smaller (i.e., more inequality) when most receive one income amount and few receive the other.

An alternative way of conceptualizing numerical inequality is to think of it as having two components: one associated with elitism of the rich and the other with isolation of the poor. Elitism of the rich might be seen as most pronounced when one income recipient is rich and the rest are poor; as high income sector enlargement proceeds, the rich become less elite. Likewise, isolation of the poor is most pronounced when one income recipient is poor and the rest are rich; isolation of the poor thus increases as high income sector enlargement proceeds. Intuitively, one might want to say that at the beginning of high income sector enlargement, the reduction of elitism of the rich dominates the increase in isolation of the poor and numerical inequality thereby decreases, whereas the reverse is true at the other end of the process. In between, an interior minimum for numerical inequality can be generated if isolation of the poor is regarded as overtaking elitism of the rich. This would arise if isolation of the poor and elitism of the rich are viewed as of comparable orders of magnitude, isolation of the poor increases at an increasing rate, and elitism of the rich decreases at a decreasing rate. Furthermore, if the two components are viewed as opposite sides of the same coin, one might justifiably conceive of numerical inequality as symmetric with minimum at the fifty-fifty point.

These lines of reasoning lead to the conclusion that numerical inequality is $U$ shaped in high income sector enlargement growth, and so too (at least in the interior) is total inequality. Once again, we have arrived at an inequality pattern that conflicts with the commonly-used indices.

\subsection{An inconsistency}

I conclude that some of the familiar measures of inequality yield rankings inconsistent with various conceptions of how inequality is changing in the process of high income sector enlargement. Many of these indices are Lorenz-consistent; this is not their problem. The problem is that the Lorenz criterion is a partial ordering, and when these indices complete the ordering, they do so in a way that is both unjustified and, in my view, unjustifiable.

To resolve the inconsistency, we might seek a criterion that satisfies two desiderata: (i) it is consistent with the Lorenz criterion, in the sense that whenever the Lorenz criterion provides a ranking, the new criterion yields the same ranking, and (ii) it generates a $U$ pattern in high income sector enlargement growth. Section 4 considers two ways that have been 
proposed for moving beyond the Lorenz ordering and concludes that neither satisfies both desiderata. Section 5 proposes an alternative that does.

\section{Results regarding generalized Lorenz curves and the axiom of composite transfers}

Recent papers by Shorrocks (1983) and by Shorrocks and Foster (1985) propose additional axioms which help complete the ordering of income distributions when the Lorenz criterion is indecisive. This section examines whether the axioms proposed are helpful for making inequality comparisons in a dualistic economy with income growth. The answer, alas, is negative.

Shorrocks (1983) addresses the question of how to rank income distributions taking account of location and dispersion aspects simultaneously. Shorrocks' recommended solution, derived axiomatically from properties of social welfare functions, is to rank income distributions according to Generalized Lorenz Curves, the GLC being a transformation of the familiar Lorenz curve with the vertical axis elongated to gauge income amounts rather than income shares. Thus, one distribution dominates another by the GLC criterion if, after ordering the recipients from lowest income to highest, the cumulative incomes of the first distribution always exceed those of the second. Shorrocks was not the first to rank income distributions according to such a criterion - I, for instance, did exactly that in my 1977 paper comparing income distributions over time in the economic growth of Brazil - but he was the first to justify this procedure axiomatically. His paper, though imaginative and careful, is not helpful in making inequality comparisons in high income sector enlargement processes. Clearly, in high income sector enlargement, the new Generalized Lorenz Curve dominates the old, and therefore the new distribution welfare-dominates the old by Shorrocks' criterion. But that is not the issue - ours is inequality comparisons - and Shorrocks (1983) does not resolve the matter, since in that work, level of income and inequality in the distribution of income are blended into a single criterion.

Shorrocks and Foster (1985) directly address the inequality comparison issue. They offer a plausible axiom drawn from the work of Sen (1973), Kolm (1976), Cowell (1977) and Kakwani (1980) and derive from it a theorem which helps make inequality comparisons when the Lorenz criterion cannot. The axiom pertains to a composite transfer of a given amount of income consisting of two parts: a progressive transfer of a large sum from a relatively rich person to a relatively poor one, and a regressive transfer of a small sum from a relatively rich person to another who is even richer. The transfersensitivity axiom weights the large gain of the poor person more than it does the small gain of the rich person. Consequently, the axiom states that such a composite transfer should be viewed as reducing inequality. From this axiom, Shorrocks and Foster derive the following theorem regarding inequality comparisons when Lorenz curves cross:

Theórem. Suppose the Lorenz curve for income distribution $X$ lies initially above that for distribution $Y$, and that the two curves then cross once. Suppose further that the variance of $\left.Y\left(\sigma_{Y}^{2}\right)\right)$ is at least as great as the variance of $X\left(\sigma_{X}^{2}\right)$. Then, the inequality of $Y$ is greater than the inequality of $X$, i.e., $\mathrm{I}(\mathrm{Y})>\mathrm{I}(\mathrm{X})$, for all inequality measures satisfying the transfer sensitivity axiom.

It may be shown that the conditions of the theorem are met in the early stages of high income sector enlargement growth. We have already seen that the Lorenz curves cross once, as depicted in fig. 1c. Consistent with Shorrocks and Foster's terminology, call the pre-enlargement Lorenz curve $X$ and the post-enlargement Lorenz curve $Y$. The variance may 
be shown to be a quadratic in the size of the modern sector, rising when the modern sector is small and falling when it is large. ${ }^{9}$ In the zone of rising variance, the conditions of Shorrocks and Foster's, theorem are met: Lorenz curve $X$ cuts Lorenz curve $Y$ from above and $\sigma_{Y}^{2}>\sigma_{\mathrm{X}}^{2}$ (In the zone of falling inequality, the variance inequality is reversed, so the theorem does not apply.)

Applying the theorem to the zone of rising variance, the conclusion of the theorem is that $I(Y)>I(X)$. That is, inequality rises with high income sector enlargement until the turning point of the variance is reached. In this way, Shorrocks and Foster's theorem provides a criterion for comparing inequalities in some situations when the Lorenz criterion is incomplete.

Unfortunately, the inequality comparison derived from Shorrocks and Foster's theorem seems to go the wrong way: their criterion has inequality rising when a small fraction of income recipients are in the high income sector and that sector expands to include more people. Intuitively, though, I see no reason that inequality should increase in this circumstance. I would want to say, contrary to their criteria, that when a small high income sector is enlarged to absorb an increased population share, inequality decreases. Thus, despite its clear attraction for ranking inequalities when redistributing a given amount of income, I conclude that the approach offered by Shorrocks and Foster is not helpful for ranking inequalities in enlargement processes in which income is growing.

\section{Toward an axiomatization of inequality in an economy with income growth}

The time has come to axiomatize inequality along the lines discussed above. It would assuredly be desirable that the axiomatization be consistent with the Lorenz axiomatization. If this were so, it would yield an identical ranking of inequality whenever the Lorenz criterion is capable of ranking income distributions. But in addition, it would rank the inequality of two income distributions in some cases when the Lorenz criterion cannot rank them because of Lorenz curve crossing. Fortunately, this is possible. The axioms I propose further complete the inequality ranking left incomplete by the Lorenz ranking, while ranking as the Lorenz criterion does whenever the Lorenz criterion produces a ranking. I begin by referring the reader to the Lorenz axioms A1-A4 on page 358: these serve as the basis for what follows.

In searching for additional axioms, I have found it useful to conceptualize the inequality in a dualistic economy as consisting of two parts: gap inequality and numerical inequality. Some observers may care more about one aspect of inequality than the other, but it would be unusual not to be concerned about both. The Lorenz criterion is as incomplete as it is, because it confounds these two logically distinct notions by amalgamating them into measures of income shares.

Gap inequality is the idea that inequality varies directly with the spread in income between persons in the high and low income groups. This idea is so widely shared as to be virtually universal. Absolute gap inequality would appropriately be

\footnotetext{
${ }^{9}$ The quadratic form follows from the limitation to two incomes $Y_{\mathrm{H}}$ and $Y_{\mathrm{L}}$, $\operatorname{var}=\frac{n_{\mathrm{H}} Y_{\mathrm{H}}^{2}+n_{\mathrm{L}} Y_{\mathrm{L}}^{2}}{n}-Y^{2}=\frac{\left(Y_{\mathrm{H}}-Y_{\mathrm{L}}\right)^{2}}{n^{2}}\left[n_{\mathrm{H}}\left(n-n_{\mathrm{H}}\right)\right]$.
}

The variance increases when the first person enters the modern sector and decreases when the last person does; the second derivative is negative. Thus there exists an initial zone in which the variance rises with high income sector enlargement. 
measured by income differences, while relative gap inequality would be measured by income ratios. Consistent with the Lorenz criterion as a basis for making relative inequality comparisons, and consistent also with the prevailing concern for changes in relative income inequality, it is the income ratio that would be relevant to consider. In the two-income, $n$ person world under analysis here, define relative gap inequality $R$ as the ratio of the per capita income of persons in the high income group $\left(\mathrm{Y}_{H}\right)$ to the per capita income of persons in the low income group $\left(\mathrm{Y}_{L}\right)$. Then: $R=Y_{H} / Y_{L}$. Defined in this way, when all incomes are the same, it is arbitrary who is high income and who is low, so $Y_{H}=Y_{L}$ in that case. I then propose:

A5. Axiom of relative gap inequality: Relative gap inequality increases/ remains the same/decreases if and only if the ratio of per capita income in the high income sector to that in the low income sector increases/remains the same/decreases.

Thus, by $\mathrm{A} 5, R(\theta)$, defined for all $\theta \geqq 1$ is specified so that $R(\theta)$ is increasing in $\theta$ and $\mathrm{R}(1)=1$.

The 'if' part of the axiom - that a change in the income ratio will change inequality in the same direction - is not new. In fact, it is implied by A4, which is why the Lorenz axioms suffice to rank inequality in the cases of high income sector enrichment and low income sector enrichment. The 'only if' part of the axiom is new. Most importantly, it states that if the income ratio between the two sectors remains the same, then gap inequality must remain unchanged. The intuitive appeal of this property is clear. Its force comes with reference to high income sector enlargement, wherein incomes per person in the two sectors, and hence their ratio, are constant, provided both sectors have strictly positive numbers of persons in them. Thus, $A 5$ implies that gap inequality is constant in high income sector enlargement. ${ }^{10}$

Numerical inequality is the idea that inequality is related to the sizes of groups in a population. It is reasonable to regard there as being a most equal numerical distribution, not necessarily unique, and to expect that the most numerically equal distribution will be located at some central point. Accordingly, define numerical inequality $N$ as $N=N\left(n_{H}, / n\right)$, where $n_{H}$ is the number of persons in the high income sector and $n$ is the total population size. $\phi=n_{H} / \mathrm{n}$ can take any rational value in the interval $[0,1]$.

Consistent with the ideas presented in section 3.3 above, I propose:

A6. Axiom of numerical inequality: Numerical equality is greatest when the population is divided evenly between two groups; numerical inequality is greater the further is the actual division from fifty-fifty. ${ }^{11}$

Thus, $N(\phi)$ is decreasing (increasing) in $\phi$ for all $\phi$ less than (greater than) 0.5 .

It need not concern us what precise metric to use to determine the distance of an actual distribution from fifty-fifty, as long as we understand that 51-49 is somewhat away, 60-40 is further away, and 99-1 is furthest away, and the measure we use has this monotonicity property in both directions. Symmetry might also be a desirable property to impose.

\footnotetext{
10 This statement needs to be qualified at the endpoints. One would want to say that gap inequality increases from zero when the first person leaves the low income sector for the high income sector, and likewise that gap inequality decreases to zero when the last low income person is absorbed into the high income sector.

${ }^{11}$ In looking at population subsamples, the most equal point might correspond to population shares other than fifty-fifty. For present purposes, the precise location of the numerically most equal point is of little consequence as long as it is in the interior of $(0,100 \%)$.
} 
As regards the three dualistic development typologies, A6 implies:

(i) Numerical inequality is unchanged in high income sector enrichment.

(ii) Numerical inequality is unchanged in low income sector enrichment.

(iii) Numerical inequality follows a $U$ pattern in high income sector enlargement. ${ }^{12}$

It is reasonable and appealing to regard overall inequality in a dualistic economy with income growth as a composite of gap inequality and numerical inequality. When strictly positive weight is given to each, term the resulting concept composite inequality. Define composite inequality as $C=C\left(R\left(Y_{H} / Y_{L}\right), N\left(n_{H} / n\right)\right)$. We need an axiom specifically relating composite inequality to gap inequality and numerical inequality. Consequently, I propose:

A7. Axiom of composite inequality: Composite inequality depends directly upon gap inequality and numerical inequality. If one of the components is unchanged, composite inequality varies directly with the level of the other. The creation/elimination of an income gap creates/eliminates composite inequality.

Thus, $\mathrm{C}$ is increasing in $R$ and $N$ and $\mathrm{C}>0$ if and only if $R>1$. Note also that this axiom permits a 'discontinuity' at the endpoints in the sense that when we go from a situation where all individuals have the same income to one where a single individual has a different income from the others, inequality may jump sharply. I view this as a strength, not a weakness, of A7.

We are now in a position to apply the preceding axioms to the three types of dualistic growth:

For high income sector enrichment:

(i) Gap inequality increases.

(ii) Numerical inequality is unchanged.

(iii) Composite inequality increases.

For low income sector enrichment:

(i) Gap inequality decreases.

(ii) Numerical inequality is unchanged.

(iii) Composite inequality decreases.

For high income sector enlargement: (see fig. 2)

(i) When the first person enters the high income sector, gap inequality increases, because people are no longer equally poor.

(ii) Gap inequality is unchanged in the interior.

(iii) When the last person enters the high income sector, gap inequality decreases, because everyone has equally

12 This statement pertains to the interior segment only, i.e., strictly positive numbers of persons are in both sectors. 
high income.

(iv) Numerical inequality falls until the fifty-fifty point, rises thereafter.

(v) Composite inequality is created when the first person enters the high income sector and is eliminated when the last person leaves the low income sector.

(vi) In the interior, composite inequality falls until the fifty-fifty point and rises thereafter.

The conclusions that composite inequality rises in high income sector enrichment and falls in low income sector enrichment are noteworthy only because something would be wrong with our axioms were the results otherwise. Much more important is the finding for high income sector

(a)
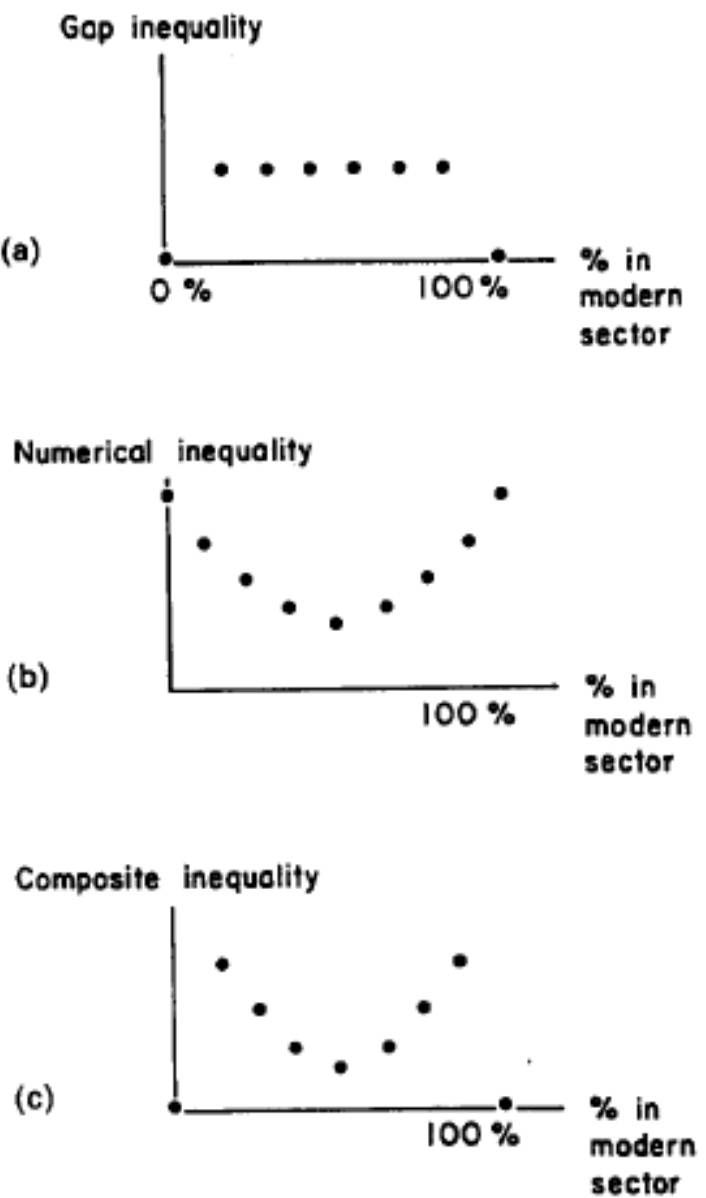

Fig. 2. Inequality change in high income sector enlargement. (a) Gap inequality in high income sector enlargement, (b) Numerical inequality in high income sector enlargement, (c) Composite inequality in high income sector enlargement.

enlargement growth: In (the interior of) high income sector enlargement, gap inequality is constant, numerical inequality is $U$-shaped, and total inequality is U-shaped. This result is so important, because no sign of an inverted-U pattern appears.

Recall that in the high income sector enlargement process many inequality indices exhibit inverted-U patterns for income inequality; these include some that are strictly Lorenz-consistent (the Gini coefficient, Theil's entropy measure, Theil's second measure, the Atkinson index and the coefficient of variation), one that is weakly Lorenz-consistent (the income share of the poorest $\mathrm{X} \%$ of the population), and one that is not Lorenz-consistent (the log variance). Thus: For the 
case of high income sector enlargement growth, the rankings obtained from many commonly-used indices are opposite from the inequality rankings that would be derived on the basis of the gap inequality and numerical inequality axioms.

\section{Indexing A1-A7}

The axiomatic set A1-A7 is best operationalized by constructing statistical measures for use by applied researchers. The process of representing axioms by a statistical measure is called indexing. Any measures so constructed should fully reflect the new axiomatization, but will also go beyond it by completing the ordering in ways implied by the measures themselves. The properties of any proposed measures need to be scrutinized with great care, so as to assure their consistency with intuitive concepts of inequality.

The first question that immediately arises is whether there exists any inequality index consistent with A1-A7. Indeed there is. Take

$$
I=\left(\frac{Y_{\mathrm{H}}}{Y_{\mathrm{L}}}-1\right)^{\alpha}\left(K+\frac{1}{4}-\frac{n_{\mathrm{H}} n_{\mathrm{L}}}{n^{2}}\right)^{1-\alpha},
$$

where

$Y_{\mathbf{H}}=$ income per capita in high income sector,

$Y_{\mathrm{L}}=$ income per capita in low income sector,

$n_{\mathrm{H}}=$ number of high income recipients,

$n_{\mathrm{L}}=$ number of low income recipients,

$n=$ total number of income recipients,

$\alpha=$ parameter ranging from zero to one,

$K=$ parameter.

This measure is obviously anonymous and hence satisfies $A 2$. It is the product of two exponentiated terms. The first, $\left(Y_{H} / Y_{L}\right.$ -1), measures relative gap inequality. It is easily seen to be consistent with the axiom of scale irrelevance (A1), the axiom of rank-preserving equalization (A3), and the axiom of relative gap inequality (A5). The second term, $\left(K+\frac{1}{4}-n_{H} n_{L} / n^{2}\right)$, measures numerical inequality. This term is derived by regarding an equal division of the population between higher and low income groups as most equal in terms of numerical inequality. At the most equal point $n_{H} / n=1 / 2$ and $n_{L} / n=1 / 2$. Using the product norm, the most equal point has equality $\frac{1}{2} * \frac{1}{2}=\frac{1}{4}$ and any other point has equality $\left(n_{H} / n\right)\left(n_{L} / n\right)$. The difference between actual equality and the most equal allocation is then $\left(n_{H} / n\right)\left(n_{L} / n\right)-1 / 4$. If this difference is subtracted from the amount of numerical inequality at the most equal point, $K$, the result may be taken as a measure of numerical inequality. Note that this component satisfies the axiom of population replication (A3) as well as the axiom of numerical inequality (A6). The exponent $\propto$ gauges the relative importance of gap inequality compared to numerical inequality. The values $\propto=$ 1 and $\propto=0$ represent concern only for gap inequality and only for numerical inequality, respectively. Intermediate values of $\propto$ strictly between 0 and 1 represent a concern for composite inequality, expressed as a positive function of gap inequality and numerical inequality, satisfying A7. Note that as long as $\propto>0$ (i.e., gap inequality receives some weight, as indeed it should for any reasonable inequality index), when incomes in .the two groups are the same, composite inequality is zero. Otherwise, it is positive. 
This shows that the axiomatic system A1-A7 is indexable. Having found one index that is consistent with the postulated axiomatic structure, a task for future research is to construct other possible measures in the two-income, $n$-person world and examine their properties.

\section{Conclusion}

This paper has analyzed how to measure changes in inequality in an economy with income growth. The preceding discussion distinguished three stylized kinds of economic growth:

1. high income sector enrichment,

2. low income sector enrichment,

3. high income sector enlargement, in which the high income sector expands and absorbs persons from the low income sector.

The two enrichment types pose no problem for assessing inequality change in the course of economic growth: for high income sector enrichment growth, inequality might reasonably be said to increase, whereas for low income sector enrichment growth, inequality might be said to decrease. These judgments are non-controversial and non-problematical.

Where problems arise is in the case of high income sector enlargement growth. In that case, the two alternative approaches have been shown in this paper to yield markedly different results:

1. The traditional inequality indices generate an inverted- $U$ pattern of inequality. That is, inequality rises in the early stages of high income sector enlargement growth and falls thereafter.

2. The new approach suggested here, based on axioms of gap inequality and numerical inequality, generates a $U$ pattern of inequality. That is, inequality falls in the early stages of high income sector enlargement growth and rises thereafter.

The discrepancy between the familiar indices and the alternative approach based on axioms of gap inequality and numerical inequality bears further scrutiny. Two courses of action are possible. One might try to axiomatize inequality in ways that generate an inverted-U pattern in high income sector enlargement growth, thereby rationalizing the continued use of the usual inequality indices with the inverted-U property. Alternatively, one might extend the line of reasoning proposed here. One obvious class of measures to consider is the CES class, of which the Cobb-Douglas form I is a special case. Other tasks that would enhance the empirical usefulness of any index derived would be to extend the analysis to account for more than two sectors and for intra-sectoral inequality.

Others might wish to pursue the first course; I am at work on the second. 


\section{References}

Ahluwalia, Montek, 1976, Inequality, poverty, and development, Journal of Development Economics.

Anand, Sudhir and S.M. Kanbur, 1984, Kuznets process and the inequality-development relationship, Mimeo. (Oxford University, Oxford).

Atkinson, A.B., 1970, On the measurement of inequality, Journal of Economic Theory.

Blackorby, C. and D. Donaldson, 1978, Measures of relative equality and their meaning in terms of social welfare, Journal of Economic Theory.

Chenery, Hollis, 1979, Structural change and development policy (Oxford University Press, Oxford).

Cowell, F.A., 1977, Measuring inequality (Allan, Oxford).

Cowell, F.A. and K. Kuga, 1981, Inequality measurement: An axiomatic approach, European Economic Review.

Fei, John C.H. and Gustav Ranis, 1964, tjevelopment of the labor surplus economy (Irwin, Homewood, IL).

Fields, Gary S., 1977, Who benefits from economic development? - A reexamination of Brazilian growth in the 1960s, American Economic Review.

Fields, Gary S., 1979, A welfare economic analysis of growth and distribution in the dual economy, Quarterly Journal of Economics.

Fields, Gary S., 1980, Poverty, inequality, and development (Cambridge University Press, Cambridge).

Fields, Gary S. and John C.H. Fei, 1978, On inequality comparisons, Econometrica.

Foster, James, 1985, Inequality measurement, in: Peyton Young, ed., Fair allocation, forthcoming.

Kakwani, Nanak, 1980, Income inequality and poverty (Oxford University Press, Oxford).

Knight, John B., 1976, Explaining income distribution in less developed countries: A framework and an agenda, Bulletin of the Oxford Institute of Economics and Statistics.

Kolm, S.-C, 1976, Unequal inequalities, Journal of Economic Theory.

Kondor, Y., 1975, Value judgements implied by the use of various measures of income inequality, Review of Income and Wealth.

Kuznets, Simon, 1955, Economic growth and income inequality, American Economic Review.

Kuznets, Simon, 1966, Modern economic growth (Yale University Press, New Haven, CT).

Lewis, W. Arthur, 1954, Economic development with unlimited supplies of labor, Manchester School.

Loehr, William and John P. Powelson, 1981, The economics of development and distribution (Harcourt Brace Jovanovich, New York).

Robinson, Sherman, 1976, A note on the U hypothesis relating income inequality and economic development, American Economic Review.

Sen, Amartya K., 1973, On economic inequality (Oxford University Press, Oxford).

Shorrocks, Anthony, 1980, The class of additively decomposable inequality measures, Econometrica.

Shorrocks, Anthony F., 1983, Ranking income distribution, Economica.

Shorrocks, Anthony F. and James Foster, 1985, Transfer sensitive inequality measures, Mimeo. (Purdue University, Lafayette, IN).

Swamy, S., 1967, Structural changes and the distribution of income by size: The case of India, Review of Income and Wealth. 